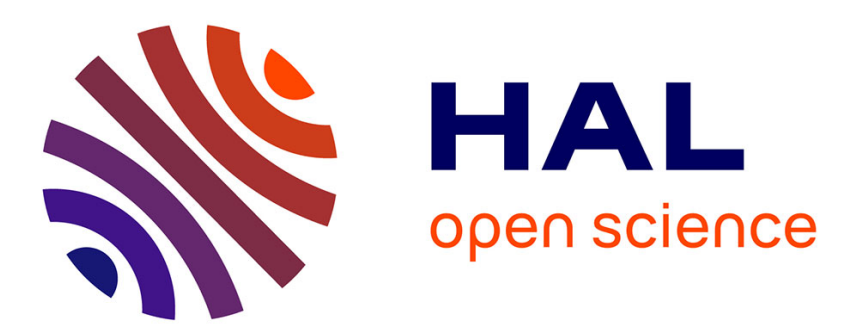

\title{
Signal-based versus nonlinear model-based damage sensitive features for delamination quantification in CFRP composites
}

Meriem Ghrib, Marc Rebillat, Nazih Mechbal, Laurent Berthe, Mikhail Guskov

\section{To cite this version:}

Meriem Ghrib, Marc Rebillat, Nazih Mechbal, Laurent Berthe, Mikhail Guskov. Signal-based versus nonlinear model-based damage sensitive features for delamination quantification in CFRP composites. International Workshop on Structural Health Monitoring, Sep 2017, Palo Alto, United States. pp.1-10. hal-01664718

\section{HAL Id: hal-01664718 \\ https://hal.science/hal-01664718}

Submitted on 15 Dec 2017

HAL is a multi-disciplinary open access archive for the deposit and dissemination of scientific research documents, whether they are published or not. The documents may come from teaching and research institutions in France or abroad, or from public or private research centers.
L'archive ouverte pluridisciplinaire HAL, est destinée au dépôt et à la diffusion de documents scientifiques de niveau recherche, publiés ou non, émanant des établissements d'enseignement et de recherche français ou étrangers, des laboratoires publics ou privés. 
Title: Signal-based versus nonlinear model-based damage sensitive features for delamination quantification in CFRP composites

\author{
Authors : Meriem Ghrib \\ Marc Rébillat \\ Nazih Mechbal \\ Laurent Berthe \\ Mikhail Guskov
}




\begin{abstract}
Structural health monitoring (SHM) is an emerging technology designed to automate the inspection process undertaken to assess the health condition of structures. The SHM process is classically decomposed into four sequential steps: detection, localization, classification, and quantification. In this paper, SHM quantification step is addressed. Particularly, we approach delamination quantification as a classification problem whereby each class corresponds to a certain damage extent. Starting from the assumption that damage causes a structure to exhibit nonlinear response, we investigate whether the use of nonlinear model based features increases classification performance. A support Vector Machine (SVM) is used to perform multi-class classification task. Two types of features are used to feed the SVM algorithm: Signal Based Features (SBF) and Nonlinear Model Based Features (NMBF). SBF are rooted in a direct use of response signals and do not consider any underlying model of the test structure. NMBF are computed based on parallel Hammerstein models which are identified with an Exponential Sine Sweep (ESS) signal. Dimensionality reduction of features vector using Principal Component Analysis (PCA) is also carried out in order to find out if it allows robustifying the quantification process suggested in this work. Experimental results on Carbon Fiber Reinforced Polymer (CFRP) composite plates equipped with piezoelectric elements and containing various delamination severities are considered for demonstration. Delamination-type damage is introduced into samples in a calibrated way using Laser Shock Wave Technique (LSWT) and more particularly symmetrical laser shock configuration. LSWT is chosen as an alternative to conventional damage generation techniques such as conventional impacts and Teflon inserts since it allows for a better calibration of damage in type, depth and size. Results show that by introducing NMBF, classification performance is improved. Furthermore, PCA allows for higher recognition rates while reducing features vector dimension.
\end{abstract}

\title{
INTRODUCTION
}

Structural Health Monitoring (SHM) combines advanced sensor technology with intelligent algorithms to interrogate the structural 'health' condition. Generally, an SHM process entails establishing: (1) the existence of damage, (2) the damage locations, (3) the types of damage, and (4) the damage severity [1]. In this work, the quantification step of the SHM process is addressed. Particularly, we approach delamination quantification as a classification problem whereby each class corresponds to a certain damage extent. Based on the assumption that in many cases damage

PIMM laboratory, Arts et Métiers ParisTech, 151 Bd de l’hôpital, 75013 Paris, France 
causes a structure to exhibit nonlinear response and that the damage monitoring process can be significantly enhanced if one takes advantage of these nonlinear effects when extracting damagesensitive features from measured data [2], we aim here at exploiting a richer nonlinear representation of our test structure and at investigating whether the use of nonlinear model based features allows for an enhanced damage quantification approach. A support Vector Machine (SVM) is used to perform multi-class classification task. Two types of features are used to feed the SVM algorithm: Signal Based Features (SBF) and Nonlinear Model Based Features (NMBF). SBF are rooted in a direct use of response signals and do not consider any underlying model of the monitored structure. NMBF are computed based on parallel Hammerstein models which are identified with an Exponential Sine Sweep (ESS) signal. Dimensionality reduction of features vector using Principal Component Analysis (PCA) is also conducted in order to find out if it allows robustifying the quantification process suggested in this work. CFRP composite coupons equipped with piezoelectric elements and containing various delamination severities are considered for demonstration. LSWT is used to introduce realistic and well calibrated delamination-type damage into test coupons.

In the following, after the presentation of laser shock wave technique and CFRP test coupons, the main key elements of the quantification workflow proposed in this paper are detailed. The experimental investigation conducted in this work is then described. Results and analysis are afterwards presented. Conclusions are given at last.

\section{TEST STRUCTURES}

Test structures considered for demonstration consist of CFRP composite plates equipped with piezoelectric elements and containing various delamination severities. Damage is introduced into samples (See Figure 2) in a calibrated way using LSWT and more particularly symmetrical laser shock configuration (See Figure 1). LSWT is chosen as an alternative to conventional damage generation techniques such as conventional impacts and Teflon inserts since it allows for a better calibration of damage in type, depth and size [3-5]. Four CFRP test coupons are considered. The first one is kept in a healthy state. The second one was subjected to a symmetrical laser impact at $0 \mathrm{~ns}$ time delay and at $100 \%$ of the maximum energy of the two laser beams [5]. This resulted in approximately $7 \mathrm{~mm}$ diameter delamination which occurred at $1.1 \mathrm{~mm}$ depth. The third coupon was subjected to two symmetrical and contiguous laser impacts which resulted in $14 \mathrm{~mm}$ diameter delamination while the fourth coupon was impacted with three contiguous impacts which in turn resulted in approximately $21 \mathrm{~mm}$ diameter delamination.
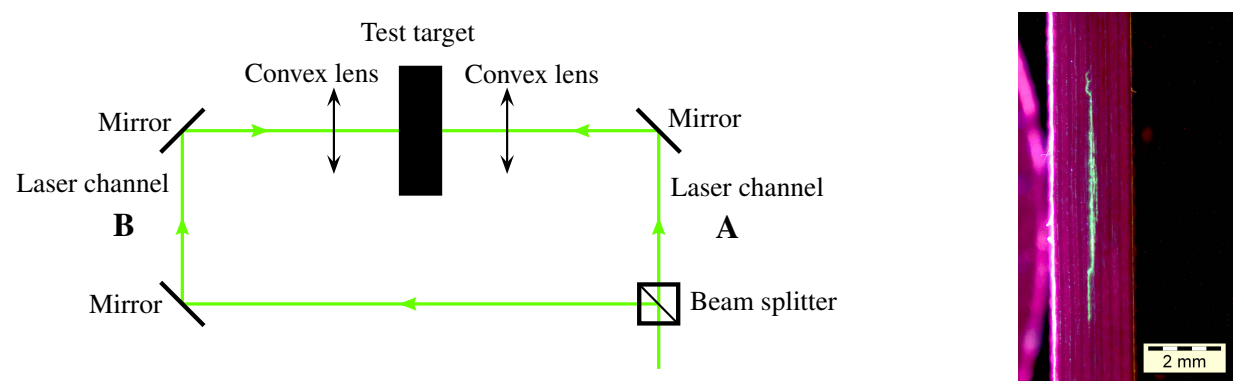

Figure 1: Experimental set-up of symmetrical Figure 2: Cross-sectional observation showlaser shock configuration ing a delamination generated using LSWT 


\section{DAMAGE QUANTIFICATION APPROACH}

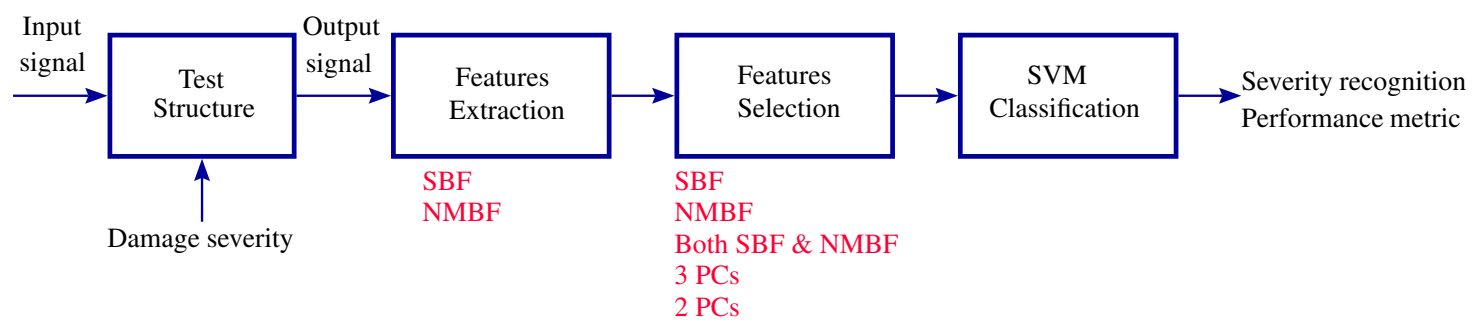

Figure 3: Workflow suggested for damage quantification

Figure 3 illustrates the main key ingredients of the quantification workflow proposed in this work. An input signal is firstly selected to excite a test structure containing a given damage severity. The structure response signal is then recorded and damage sensitive features are extracted. In this work, we investigate whether NMBF allow for an enhanced damage quantification strategy. Two types of features are thus considered: SBF and NMBF.

\section{Signal Based Features}

Signal Based Features are rooted in a direct use of response signals and do not consider any underlying model of the test structure. Four signal based features are considered in this study and are computed as follows. Let $s_{r e f}(t)$ and $s_{d}(t)$ be the structure output signal in reference and damaged state respectively, where $t$ refers to time, one defines:

TABLE I: SIGNAL BASED FEATURES.

\begin{tabular}{|c|c|c|}
\hline Damage-sensitive feature & & Details \\
\hline$C C=1-\frac{\operatorname{cov}\left(s_{r e f}(t), s_{d}(t)\right)}{\sigma_{s_{r e f}(t)} \sigma_{s_{d}(t)}}$ & (1) & $\begin{array}{l}\text { - Feature based on cross correlation } \\
\text { - } \operatorname{cov}\left(s_{r e f}(t), s_{d}(t)\right) \text { is the covariance of } \\
s_{r e f}(t) \text { and } s_{d}(t) \\
\text { - } \sigma_{s_{r e f}}(t) \text { and } \sigma_{s_{d}(t)} \text { are the standard devi- } \\
\text { ations of } s_{r e f}(t) \text { and } s_{d}(t) \text { respectively }\end{array}$ \\
\hline$N R E=\frac{\sum_{t=T_{1}}^{T_{2}}\left(s_{r e f}(t)-s_{d}(t)\right)^{2}}{\sum_{t=T_{1}}^{T_{2}} s_{r e f}(t)^{2}}$ & (2) & $\begin{array}{l}\text { - Normalized Residual Energy } \\
\text { - }\left[T_{1}, T_{2}\right] \text { is the time interval in which } \\
\text { signals of interest are analyzed }\end{array}$ \\
\hline$M A=\frac{\max _{t}\left(\left|s_{r e f}(t)-s_{d}(t)\right|\right)}{\max _{t}\left|s_{r e f}(t)\right|}$ & (3) & - Maximum Amplitude \\
\hline$E N V=\sqrt{\frac{\sum_{t=T_{1}}^{T_{2}} A_{s_{r e f, d}(t)}^{2}}{\sum_{t=T_{1}}^{T_{2}} A_{s_{r e f}(t)}^{2}}}$ & (4) & $\begin{array}{l}\text { - Signal envelope or instant amplitude } \\
\text { energy } \\
\text { - } s_{r e f, d}(t)=s_{r e f}(t)-s_{d}(t) \\
\text { - } A_{s(t)}=\sqrt{s^{2}(t)-\mathcal{H}\{s\}(t)^{2}} \\
\text { - } \mathcal{H}\{s\}(t) \text { is the Hilbert transform of } \\
\\
s(t)\end{array}$ \\
\hline
\end{tabular}




\section{Nonlinear Model Based Features}

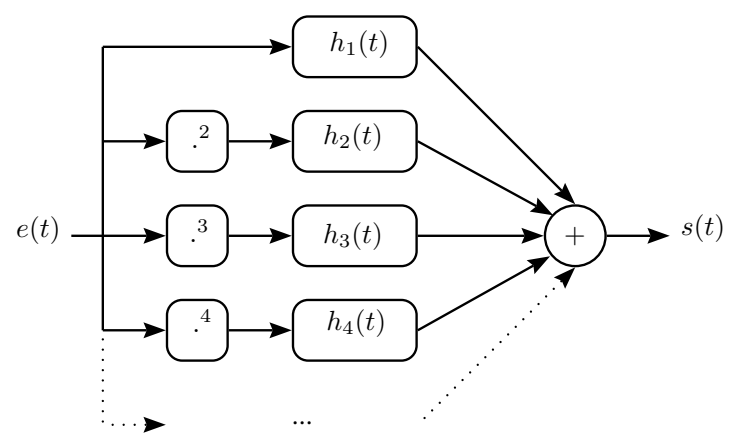

Figure 4: Parallel Hammerstein models

NMBF are considered based on previous work presented in [6,7]. Parallel Hammerstein models are used to model the damaged structure (See Figure 4). The model is fully represented by its kernels $\left\{h_{n}(t)\right\}_{n \in\{1 \ldots N\}}$ (N being the model order and can be automatically estimated [8]). The model is identified by means of Exponential Sine Sweeps excitation signal $(e(t))$. The system output $s(t)$ can be rewritten as follows:

$$
\begin{aligned}
s(t) & =\sum_{n=1}^{N}\left(h_{n} * e^{n}\right)(t) \\
& =\left(h_{1} * e\right)(t)+\sum_{n=2}^{N}\left(h_{n} * e^{n}\right)(t) \\
& =s^{L}(t)+s^{N L}(t) \\
& =s^{L}(t)+\sum_{n \text { odd }}\left(h_{n} * e^{n}\right)(t)+\sum_{n \text { even }}\left(h_{n} * e^{n}\right)(t) \\
& =s^{L}(t)+s_{o}^{N L}(t)+s_{e}^{N L}(t)
\end{aligned}
$$

The output signal is decomposed into a linear part and a nonlinear part (6). The nonlinear part is in turn decomposed into odd harmonics contribution and even harmonics contribution (8). Three features are chosen and computed as detailed in Table II.

\section{SVM and PCA}

SVMs SVM learning technique is used for the classification step. SVMs [9] are originally introduced by Vapnik and co-workers $[10,11]$ and successfully extended by a number of other researchers. SVMs are applicable to both classification and regression. When used for classification, SVMs separate a given set of binary labeled training data with a hyper-plane that is maximally distant from them (known as the maximal margin hyper-plane). For cases in which no linear separation is possible, they can work in combination with the technique of 'kernels', that automatically realizes a non-linear mapping to a feature space. The hyper-plane found by the SVM in the feature space corresponds to a non-linear decision boundary in the input space. To extend SVMs to multi-class scenario, a typical conventional way is to decompose a multi-class problem into a series of two-class problems. One can distinguish between two implementations:

- One Against All 'OAA' approach

- One Against One 'OAO' approach 
TABLE II: NONLINEAR MODEL BASED FEATURES.

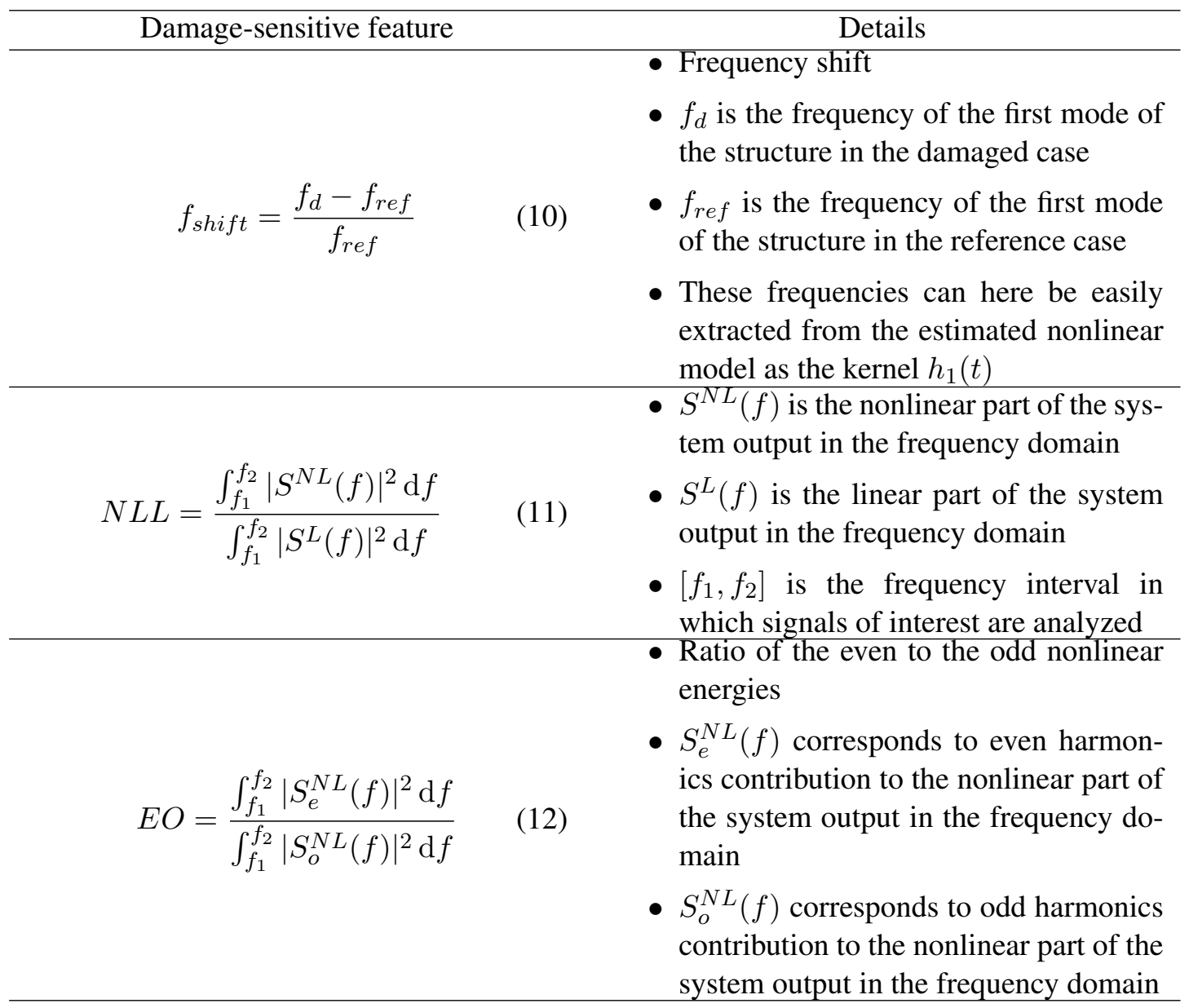

The 'OAO' and the 'OAA' are two popular strategies for multi-class SVM. 'OAO' builds one SVM for each pair of classes while 'OAA' consists of building one SVM per class, trained to distinguish the samples in a single class from the samples in all remaining classes. In this work, a Gaussian kernel SVM is considered. SVM and Kernel Methods (SVM-KM) Matlab toolbox [12] is used to perform multiclass classification.

PCA Principal Component analysis (PCA) [13] is a popular tool for linear dimensionality reduction and feature extraction. Intuitively, PCA can supply the user with a lower-dimensional picture of data when viewed from its most informative viewpoint. Several extensions of the standard PCA have been proposed such as the Kernel PCA which is the nonlinear form of PCA and which better exploits the complicated spatial structure of high-dimensional features. In this work we opted for the standard PCA since our features vector is not very high-dimensional.

\section{Input Features Scenarios}

Several input features scenarios are considered according to which features to select to feed and train the SVM algorithm:

- Scenario 1 Only SBF are used to train the SVM algorithm

- Scenario 2 Only NMBF are used to train the SVM algorithm

- Scenario 3 Both SBF and NMBF are used to train the SVM algorithm 
- Scenario 4 PCA is performed on both SBF and NMBF and only 2 principal components are used to train the SVM algorithm

- Scenario 5 PCA is performed on both SBF and NMBF and only 3 principal components are used to train the SVM algorithm

\section{EXPERIMENTAL INVESTIGATION}

Test coupons were excited using an exponential sine sweep signal with $1 \mathrm{kHz}$ minimum frequency $\left(f_{\min }\right), 100 \mathrm{kHz}$ maximum frequency $\left(f_{\max }\right), 0.45 \mathrm{~s}$ sweep duration, $10 \mathrm{~V}$ amplitude and $10 \mathrm{MHz}$ input sampling frequency. Twenty repetitions were considered. At each repetition one goes around all PZT elements and only one PZT operates as an actuator while others operate as sensors. Acquisition frequency was set to $1 \mathrm{MHz}$. An instance of measurements corresponding to a given repetition is illustrated in Figure 5.
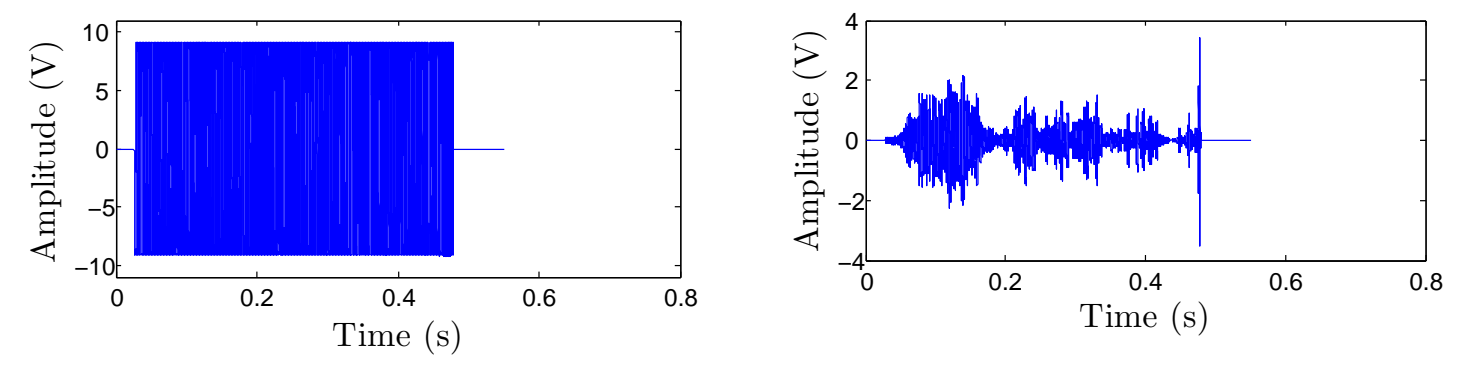

Figure 5: Left: Excitation ESS signal, Right: Structure response signal

\section{RESULTS AND ANALYSIS}

\section{From Output Signals to Input Features}

Once signals are acquired, we proceeded to information condensation, that is, to damagesensitive features computing. Each class of damage state (0: healthy, 1: one laser impact, 2: two contiguous laser impacts and 3: three contiguous laser impacts) has 400 instances. Each instance is characterized by a total of seven features. Stratified sampling is considered; $70 \%$ of data is used for training the SVM model and $30 \%$ of data is used for testing the model. In the following figures, we represent training data in SBF space, NMBF space and in principal components spaces. The first three principal components retain $94 \%$ of data variance while the first two principal components retain $78 \%$ of data variance. Figures 6 and 7 reveal a structure of four classes. Each corresponds to a state of damage severity. However, class separability is much better in NMBF space and in principal components spaces than in SBF space. 

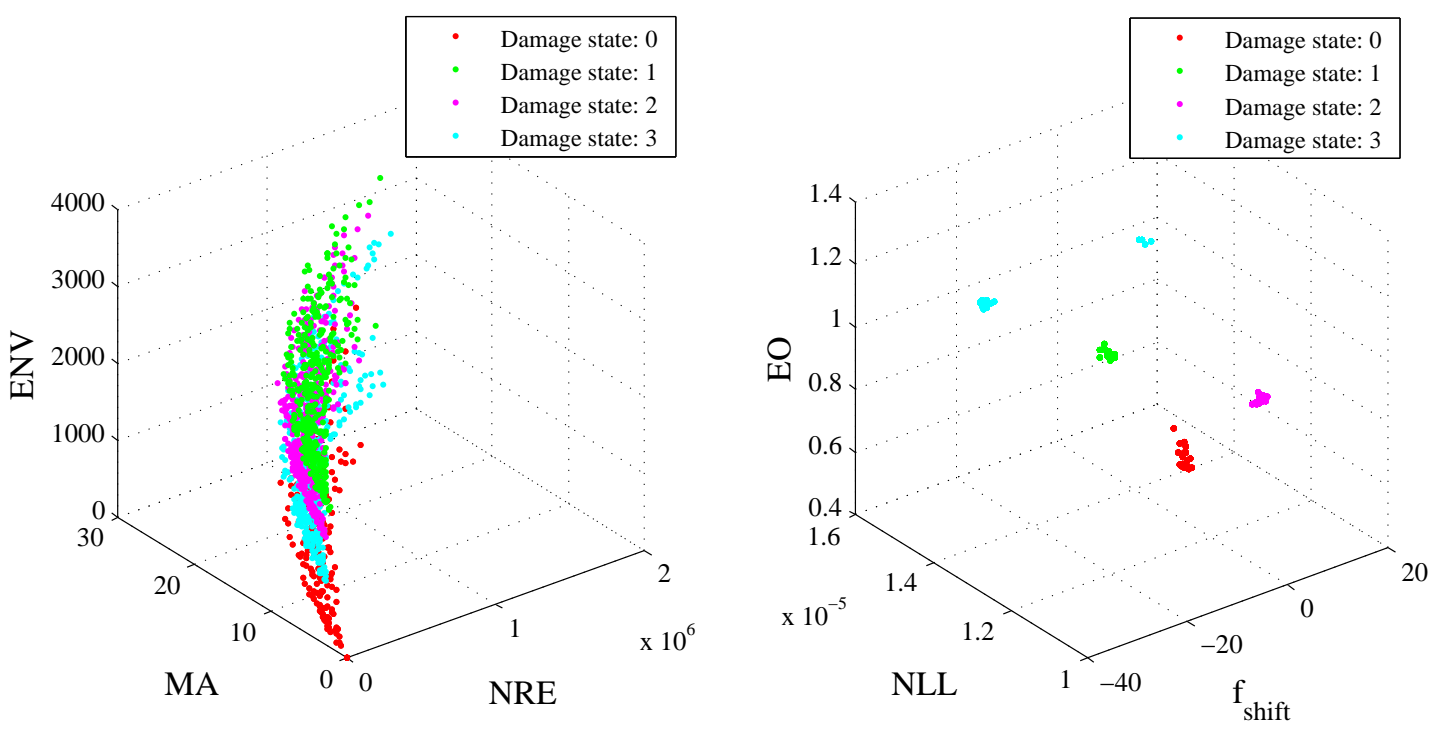

Figure 6: Data representation - Left: in SBF space, Right in NMBF space
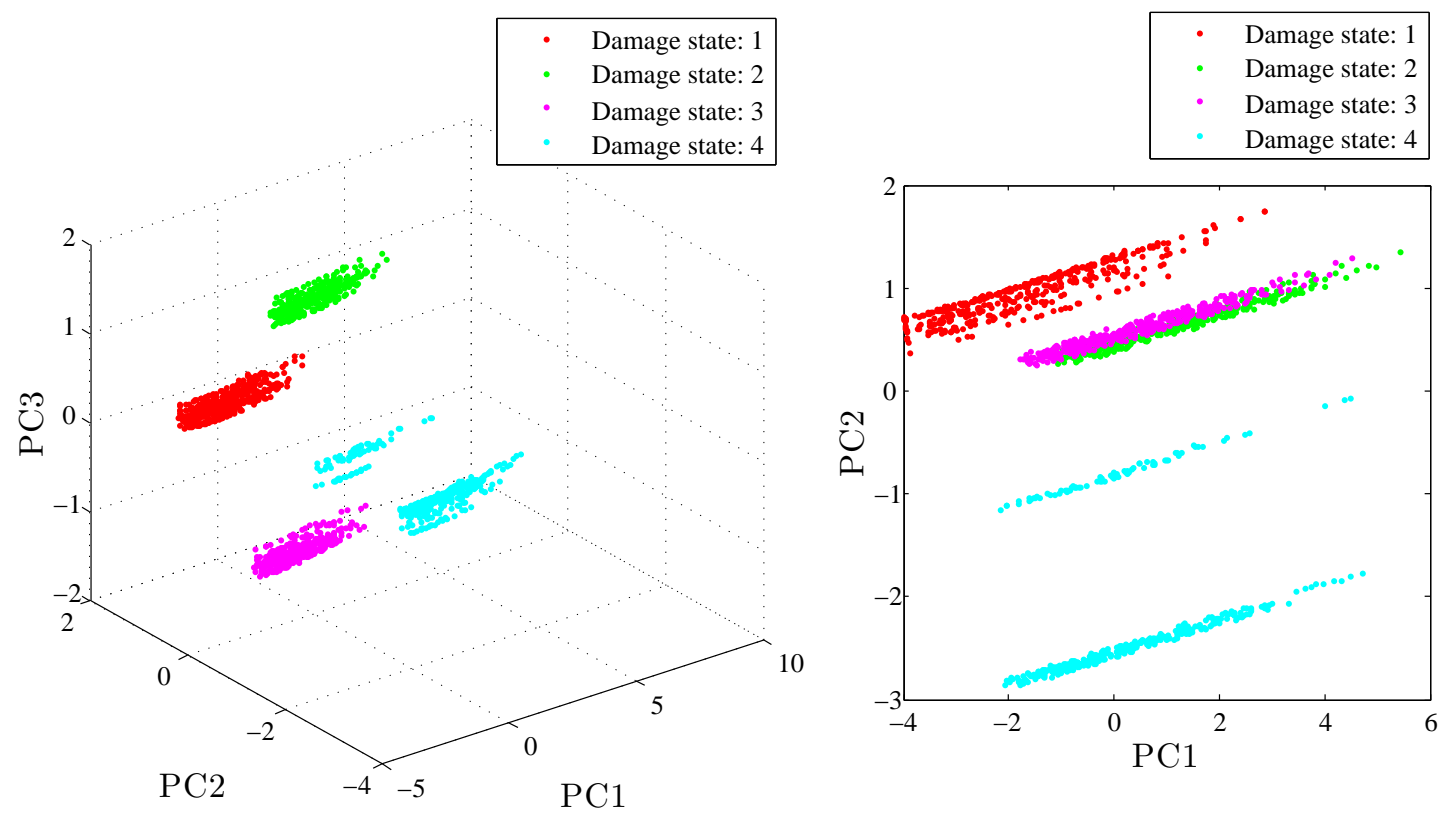

Figure 7: Data representation in principal components spaces

\section{Classification Performance}

Ten SVM models are established according to which approach is adopted ('OAO' or 'OAA') and to which features are chosen as inputs (SBF, NMBF, SBF \& NMBF, 3 PCs or 2 PCs). Models performance on test data is then assessed. As illustrated in figure 8, independently of which approach is used, SVM models trained on NMBF or on principal components perform better than those trained on only SBF. This is clearly due to class overlapping introduced by SBF and which induces the classifiers into error. 


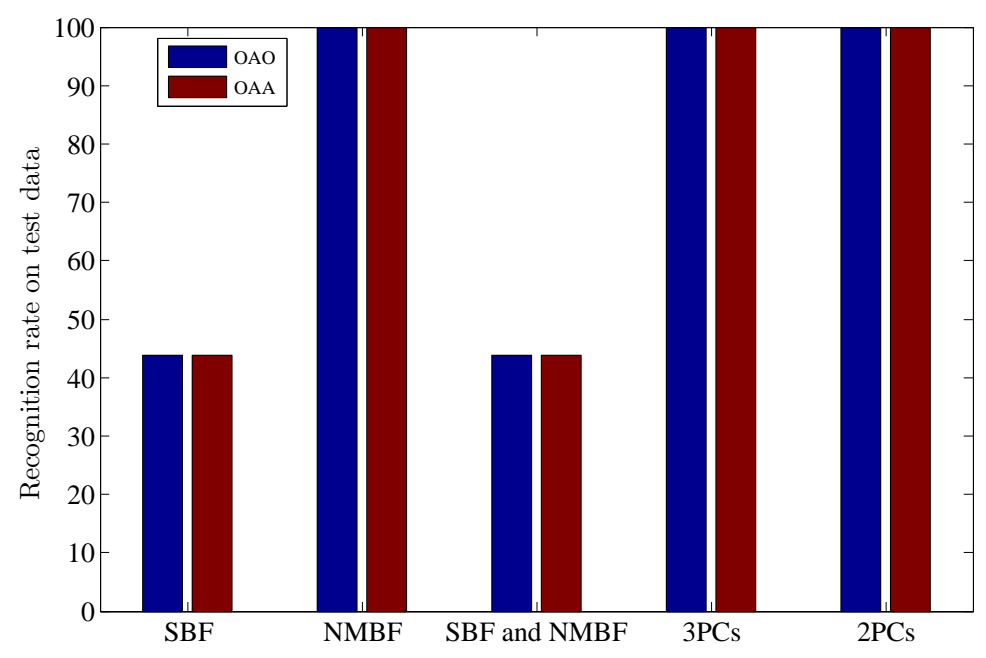

Figure 8: Performance assessment of the considered SVM models on test data

\section{CONCLUDING REMARKS}

From the outcome of our investigation one can conclude that:

- NMBF bring forward more interesting information in terms of sensitivity to damage severity

- PCA reduces features vector dimension while performing well on damage severity recognition

In our future research we intend to conduct further investigation, through numerical simulations, in order to reveal the physical phenomena behind NMBF and to better understand damage/ excitation interaction.

\section{REFERENCES}

1. Rytter, A. 1993. Vibrational Based Inspection of Civil Engineering Structures, Ph.D. thesis, Denmark.

2. Worden, K., C. R. Farrar, J. Haywood, and M. Todd. 2008. "A review of nonlinear dynamics applications to structural health monitoring," Structural Control and Health Monitoring, 15(4):540-567.

3. Gay, É., L. Berthe, M. Boustié, M. Arrigoni, and M. Trombini. 2014. "Study of the response of CFRP composite laminates to a laser-induced shock," Composites Part B: Engineering, 64:108-115.

4. Ghrib, M., L. Berthe, M. Rébillat, N. Mechbal, M. Guskov, and R. Ecault. 2016. "Laser shock a novel way to generate calibrated delamination in composites: concept and first results," in 8th European Workshop On Structural Health Monitoring, Bilbao, Spain.

5. Ghrib, M., L. Berthe, N. Mechbal, and M. Rébillat and Mikhail Guskov and Romain Ecault and Nas Bedreddine. 2017. "Generation of controlled delaminations in composites using symmetrical laser shock configuration," Composite Structures, 171:286-297.

6. Bakir, M., M. Rébillat, and N. Mechbal. 2015. "Damage type classification based on structures nonlinear dynamical signature," in 9th IFAC symposium on Fault Detection, Supervision and Safety of Technical Processes, Paris, pp. 652-657.

7. Rébillat, M., R. Hajrya, and N. Mechbal. 2013. "Detection of structural damage using the exponential sine sweep method," in International Workshop on Structural Health Monitoring, pp. 1226-2013.

8. Rébillat, M., K. Ege, M. Gallo, and J. Antoni. 2016. "Repeated exponential sine sweeps for the autonomous estimation of nonlinearities and bootstrap assessment of uncertainties," Proceedings 
of the Institution of Mechanical Engineers, Part C: Journal of Mechanical Engineering Science, 230(6):1007-1018.

9. Cristianini, N. and J. Shawe-Taylor. 2000. An Introduction to Support Vector Machines: And Other Kernel-based Learning Methods, Cambridge University Press.

10. Boser, B. E., I. M. Guyon, and V. N. Vapnik. 1992. "A Training Algorithm for Optimal Margin Classifiers," in Proceedings of the Fifth Annual Workshop on Computational Learning Theory, ACM, New York, NY, USA, pp. 144-152.

11. Vapnik, V. N. 1998. Statistical Learning Theory, Wiley-Interscience.

12. Canu, S., Y. Grandvalet, V. Guigue, and A. Rakotomamonjy. 2005, "SVM and Kernel Methods Matlab Toolbox," http://asi.insa-rouen.fr.

13. Jolliffe, I. 1986. Principal Component Analysis, Springer Verlag. 


\section{DEStech Publications, Inc.}

\section{CONTRIBUTING AUTHOR COPYRIGHT RELEASE FORM}

As author of the chapter/contribution titled Signal-based versus nonlinear model-based damage sensitive features for delamination quantification in CFRP composites, to appear in the Proceedings of Structural Health Monitoring 2017, I hereby agree to the following:

1. To grant to DEStech Publications, Inc., 439 North Duke Street, Lancaster, PA, 17602, copyright of the above named chapter/contribution (for U.S.

Government employees to the extent transferable), in print, electronic, and online formats. However, the undersigned reserve the following:

a. All proprietary rights other than copyright, such as patent rights.

b. The right to use all or part of this article in future works.

DEStech Publications thereby retains full and exclusive right to publish, market, and sell this material in any and all editions, in the English language or otherwise.

1 I warrant to DEStech Publications, Inc., that I am the (an) author of the above-named chapter/contribution and that I am the (a) copyright holder of the above-named chapter/contribution granted to DEStech Publications, Inc.

2 I warrant that, where necessary and required, I have obtained written permission for the use of any and all copyrighted materials used in the abovenamed chapter/contribution. I understand that I am responsible for all costs of gaining written permission for use of copyrighted materials.

3 I agree to assume full liability to DEStech Publications, Inc. and its licensee, and to hold DEStech Publications, Inc. harmless for any claim or suit filed against DEStech Publications, Inc. for violation of copyrighted material used in the abovenamed contribution.

Please sign and date this form and retain a copy for your records. Please include original form with your chapter/paper.

Thank you for your cooperation.

Please print name: Meriem Ghrib

Signed: Dated: 14 Mai 2017

439 NORTH DUKE STREET • LANCASTER, PENNSYLVANIA 17602-4967, U.S.A. Toll Free: (866) 401-4337 • Tel: (717) 290-1660 • Fax: (717) 509-6100 E-mail: info@destechpub.com • Internet address: www.destechpub.com 